\title{
ФЕНОМЕН «УХОДА В СВОИ МЫСЛИ» И ЕГО МЕСТО В КОНТИНУУМЕ СОЗНАНИЯ
}

\author{
А.А. ЛАПИНА, Б.В. ЧЕРНЫШЕВ
}

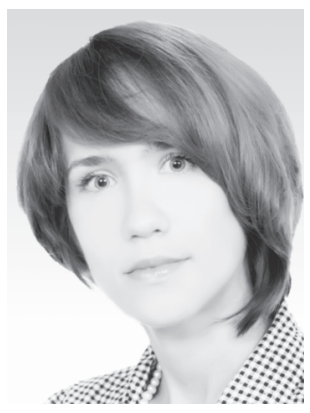

Лапина Анна Андреевна - стажер-исследователь научно-учебной группы когнитивной психофизиологии НИУ ВШЭ, аспирант департамента психологии НИУ ВШЭ.

Контакты: alapina@hse.ru

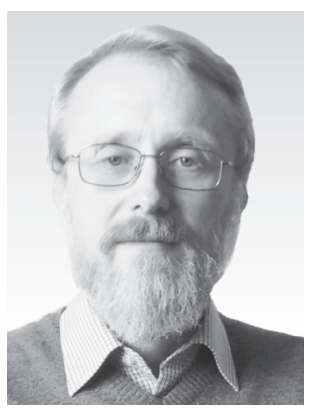

Чернышев Борис Владимирович - заведующий лабораторией когнитивной психофизиологии НИУ ВШЭ, доцент кафедры высшей нервной деятельности биологического факультета Московского государственного университета имени М.В. Ломоносова, кандидат биологических наук, доцент.

Контакты: bchernyshev@hse.ru

\section{Резюме}

Феномен «ухода в свои мысли» (англ. термин «mind-wandering») - это особое состояние сознания, связанное с полным или частичным отключением внимания от восприятия внешней сенсорной информации и его переключением на внутренние ментальные процессы. Данное состояние характеризуется высокой спонтанностью и высокой непроизвольностью в отношении своего содержания. При данном состоянии в сознании возникают мысли, которые генерируются эндогенной ментальной активностью и концентрируются вокруг значимых для личности целей, но при этом негативно интерферируют с обработкой наличной информации, повышая риск возникновения «сбоев внимания» и ошибок в текущей деятельности. С явлением «ухода в свои мысли» связана мечтательность как черта личности. Переходы в состояние «ухода в свои мысли» связаны с конкуренцией между мотивационной значимостью данного процесса в сравнении с мотивационной значимостью текущей эксплицитной деятельности и зависят от процессов когнитивного (исполнительного) контроля. Мотивационная значимость «ухода в свои мысли» определяется не 
только личностной значимостью конкретного содержания мыслей; данное состояние, видимо, входит в число неотъемлемых потребностей человека, так как обеспечивает анализ прошлого опыта и планирование будущих действий и тем самым является адаптивным приобретением человеческой психики. Феномен «ухода в свои мысли» тесно связан с рядом других психических явлений, таких как рефлексия, метапознание (метасознание) и полнота осознания («mindfulness»). В рамках настоящей статьи вводится подход, позволяющий разделить указанные процессы и обозначить особый статус «ухода в свои мысли» по признаку негативного влияния на текущую деятельность.

Ключевые слова: сознание, континуум сознания, внимание, сбои внимания, уход в свои мысли, мечтательность, рефлексия, метапознание, метасознание, полнота осознания.

\section{Введение}

Изучение содержания сознания привлекает внимание мыслителей на протяжении уже не одного века, однако по-прежнему остается одной из центральных загадок как психологии, так и науки в целом. У. Джеймс в конце XIX в. впервые описал «поток сознания» (James, 1983). В рамках данного понятия подразумевалось, что индивидуальное сознание является континуумом, в котором процесс мышления непрерывен. В поддержании «потока сознания» основная роль отводится мыслям, происхождение которых не всегда можно однозначно соотнести с внешними влияниями окружающей среды. В центре феномена «ухода в свои мысли», которому посвящена настоящая статья, лежит способность мозга самостоятельно производить и поддерживать в сознании внутренний поток информации, не имеющий явной связи с внешней стимуляцией. Изучение данного феномена имеет большое теоретическое значение для понимания состояний сознания, в которых когнитивные процессы оторваны от восприятия действительности в реальном времени.
Внимание является одним из важнейших компонентов повседневной деятельности человека, при решении важных профессиональных задач. С точки зрения П.Я. Гальперина (1998), внимание - это самостоятельный активный психический процесс, целенаправленная деятельность контроля, которая подвержена действию внешних и внутренних факторов (помех). Одним из важнейших внутренних факторов, вмешивающихся в процесс внимания и понижающих его эффективность в отношении текущей деятельности, как раз и является «уход в свои мысли».

К изучению спонтанного мыслительного процесса обращаются в течение уже нескольких веков специалисты в области психоанализа, клинической психологии, нейронауки. В нем отражаются различные характеристики личности, такие как представление о себе и о других, установки, скрытые мотивы поведения (Singer, 1993). С «уходом в свои мысли» связана мечтательность как черта личности (McMillan et al., 2013; Singer, Antrobus, 1963). Мечты, фантазии, галлюцинаторные образы возникают при сенсорной депривации, клинической патологии и 
состоянии сниженного бодрствования (Vaitl et al., 2005). Однако мечтательность отнюдь не ограничивается этими состояниями. Склонность к мечтательности у здорового человека проявляется в частоте появления эпизодов «ухода в свои мысли». Это состояние сознания возникает при любой активности на протяжении всего периода бодрствования в течение суток. На вероятность появления эпизодов «ухода в свои мысли» влияет монотонность и степень автоматизации действий в текущей деятельности, а также психофизиологическое состояние человека; например, тревога перед предстоящей важной встречей повышает частоту эпизодов «ухода в свои мысли» (Singer, 1993).

Экспериментальные исследования мечтательности и «ухода в свои мысли» начали проводиться в середине XX в. Дж.Л. Сингером и Дж.С. Антробусом (Antrobus et al., 1966; Singer, 1966). Современные исследователи в области нейронаук применяют комплексный подход для изучения «ухода в свои мысли». Большое внимание уделяется субъективным самоотчетам и проверке их достоверности с использованием поведенческих показателей при выполнении задач, требующих длительного поддержания внимания к внешним стимулам. С этой целью Дж. Смолвудом и Дж.У. Скулером в большой серии работ была использована задача «устойчивого внимания к ответу» (Sustained Attention to Response Task, SART), которая чувствительна к ошибкам внимания и эпизодам ухода в свои мысли (см., например: Smallwood, Schooler, 2006).
Чрезвычайно актуальна, но на настоящий момент явно недостаточно изучена связь «ухода в свои мысли» c другими феноменами сознания, такими как полнота осознания (mindfulness), рефлексия и метапознание (метасознание). Ниже мы подробно рассмотрим взаимосвязь этих понятий и явлений и определим специфику «ухода в свои мысли» как одного из важнейших состояний в континууме сознания.

\section{«Уход в свои мысли» как состояние сознания}

Внутри континуума сознания расположено относительно устойчивое специфическое содержание сознания, которое может изменяться во времени по ряду характеристик. В частности, суточные колебания уровня бодрствования и активации сопровождаются изменением содержания сознания (Vaitl et al., 2005). Состояния сознания в континууме сна-бодрствования и степени осознанного контроля включают в себя: активное деятельное бодрствование, «уход в свои мысли» на фоне бодрствования, гипнагогическое состояние на фоне засыпания и сновидения во время сна.

Под термином «уход в свои мысли» (mind-wandering) мы понимаем состояние сознания, которое, по некоторым оценкам, занимает примерно половину времени в период бодрствования (Killingsworth, Gilbert, 2010). Данное состояние связано с полным или частичным отсоединением внимания от восприятия внешней информации. Оно характеризуется высокой спонтанностью (возникает вне прямой 
зависимости от внешних стимулов, хотя может косвенно провоцироваться ими по цепочке ассоциаций), высокой непроизвольностью (Schad et al., 2012; Singer, 1993; Smallwood, 2013). В данном состоянии в сознании возникают мысли, которые генерируются эндогенно и негативно интерферируют с обработкой информации, которая необходима для качественного выполнения текущих задач, требующих концентрации и направленности внимания (Smallwood, 2013). Рациональность мышления в эпизодах «ухода в свои мысли» низкая, но все же выше, чем в сновидениях, преобладает мышление в образах (Wolman, Kozmova, 2007). Эпизоды «ухода в свои мысли» возникают чаще всего непроизвольно. Однако в ряде случаев они появляются по воле человека, например, при понижении интереса к происходящему вокруг, сексуальных фантазиях (Singer, Antrobus, 1963).

Обобщая имеющиеся в литературе данные, касающиеся «ухода в свои мысли», можно выделить следующие основные характеристики этого феномена:

1) отсоединение внимания (decoupling) от внешней текущей деятельности и перенаправление фокуса внимания на внутренний эндогенно сгенерированный поток информации;

2) понижение уровня контроля над содержанием сознания;

3) когнитивные процессы переключаются на цели, связанные с личными эмоционально окрашенными мотивами, установками, целями, отношениями, происходит активное извлечение информации из эпизодической памяти.
Приведем в качестве примера два крайних вида проявления «ухода в свои мысли» - руминации и инкубация творческих решений.

Руминации, или повторяющиеся, навязчивые мысли, - это одна из форм «ухода в свои мысли», нередко связанная с депрессивными, маниакальными тенденциями или повышенной тревожностью (Watkins, 2008). По определению Л.Л. Мартина и А. Тесcеpa (Martin, Tesser, 1996), повторяющиеся мысли - это часть сознательного мыслительного потока, который вращается вокруг общей, играющей важную роль для человека темы при отсутствии непосредственных внешних задач, требующих мыслительной деятельности.

Согласно теории стиля реагирования Е.Р. Уоткинса (Watkins, 2008), умственная «жвачка» возникает в ответ на депрессивное настроение и является стабильной характеристикой индивида. Эта пассивная стратегия решения проблем, уходящая своими корнями в детство, является негативным последствием гиперопеки и повышенной критики. В случаях выраженного стресса (особенно при посттравматическом стрессорном расстройстве) руминации сами по себе могут стать существенным фактором застойности данного патологического состояния и требуют психотерапевтического вмешательства (Speckens et al., 2007).

C другой стороны, состояние «ухода в свои мысли» может обеспечивать инкубацию креативных решений - опять же при условии личностной значимости; это состояние может проявляться как «профессорская рассеянность», «поэтическая 
рассеянность» и т.п. Связь частоты грез наяву и «ухода в свои мысли» с креативностью была показана в ряде исследований с использованием Теста необычного использования (Unusual Uses Task, UUT) и в тестах на креативность вербальной экспрессии (Singer, McCraven, 1961; Baird et al., 2012). Также была показана связь частоты «ухода в свои мысли» с принятием экономически выгодных решений (Smallwood, 2013).

После того как еще в XIX в. У. Джеймс интроспективно исследовал феноменологию образов и мыслей, исследования спонтанных мыслительных процессов велись в рамках психоаналитического подхода с использованием интроспекции и метода свободных ассоциаций (James, 1983; Rapaport, 1957). В середине прошлого века на изучении грез наяву и мечтательности фокусировались исследования Дж.Л. Сингера, Дж.С. Антробуса с сотрудниками (Antrobus et al., 1966; Singer, McCraven, 1961).

\section{«Уход в свои мысли» и мечтательность}

Феномен «ухода в свои мысли» тесно связан с мечтательностью, которая рассматривается как личностная черта, определяющая склонность уходить в свои мысли и фантазии (Singer, Antrobus, 1963). С точки зрения сенсорно-тонической теории У. Вернера (Werner, Wapner, 1949), в ходе индивидуального развития склонность к грезам наяву может формироваться в раннем детстве под влиянием подкрепляющих родительских воздействий и социокультурной среды, в которой находится ребенок (Singer, Schonbar, 1961). Уход в свои фантазии, мечты выступает как способ совладания с задержкой в реализации намерения, действия или потребности и может проявляться в виде проигрывания перед внутренним взором сценариев событий, не успевших реализоваться на текущий момент или потенциально неосуществимых. Объединяя большое количество данных клинических интервью, самоотчетов, результатов методик, связанных с различными личностными переменными, и проведя факторный анализ, Дж.Л. Сингер и Дж.С. Антробус определили связь мечтательности с определенными чертами личности (Singer, Antrobus, 1963). Была создана методика оценки эпизодов мечтательности (The Imaginal Processes Inventory, IPI) (Singer, Antrobus, 1966). Факторным анализом было выделено три кластера - стиля мечтательности:

1) кластер внимания (недостаточный контроль внимания) - приятные фантазии, проигрывание будущих событий, сексуальное возбуждение, самостимулирование при монотонной деятельности;

2) кластер тревожности (стиль, связанный с виной-дисфорией) страх, агрессия в мечтах, депрессивные руминации, ориентация в прошлое;

3) кластер любопытства (позитивно-конструктивный стиль) фантазии используются творчески, отмечается стремление к компетентности, исследовательское поведение.

Правомерность данного разделения на индивидуальные стили подтверждалась в течение 40 лет на разных возрастных, этнических, клинических и т.д. выборках (McMillan et 
al., 2013). Т. Жиян и Дж.Л. Сингер обнаружили связь трех стилей мечтательности с отдельными чертами характера по Пятифакторной методике (Zhiyan, Singer, 1997). Позитивно-конструктивный стиль положительно коррелирует с результатами по шкале «Открытость опыту», отражает любознательность, открытость новизне, креативность в использовании образов, идей в поведении. Плохой контроль внимания связан с низкими показателями по шкале «Добросовестность». Стиль, связанный с виной-дисфорией, коррелирует положительно со шкалой «Нейротизм». Все это указывает на немаловажную роль мечтательности и «ухода в свои мысли» в структуре личности.

\section{«Уход в свои мысли» в контексте мотивации и аффекта}

И. Клингер в своих исследованиях постулирует, что относительная важность для личности текущих проблем определяет материал, который наполняет эпизоды «ухода в свои мысли» (Klinger et al., 1973). Связь аффекта, мотивации, мышления была показана неоднократно различными психологическими школами. Мысль представляет собой «потребностно-эмоционально-содержательную субстанцию» (Шадриков, 2014, с. 32). Л.С. Выготский, указывая на единство аффекта и интеллекта, писал: «...во всякой идее содержится в переработанном виде аффективное отношение человека к действительности, представленной в этой идее» (Выготский, 1982, с. 21-22). Многочисленные современные исследования эмоций показывают их влияние на память и внимание (Kaplan et al., 2012). Таким образом, аффекты усиливают и подкрепляют мотивационное поведение и организуют содержание памяти и избирательность внимания, тем самым регулируя содержание нашего сознания (Rapaport, 1951; Kaplan et al., 2012). Интенсивный эмоциональный опыт до выполнения задачи на устойчивое внимание в лабораторных условиях увеличивает частоту эпизодов «ухода в свои мысли» (Singer, 1993).

Теория стиля реагирования Е.Р. Уоткинса (Watkins, 2008) предполагает, что человек воспринимает свое текущее состояние и поведение, а затем сопоставляет все это с важными опорными точками - целями, стандартами или желаемым результатом. Обнаруженное несоответствие между фактическим состоянием и эталоном, например важной для личности целью, запускает автоматические размышления, которые должны привести к коррекции поведения. Уход в свои мысли может способствовать поиску альтернативных способов достижения необходимых конечных результатов. В качестве эталонов могут выступать внутренние стимулы (настроение, чувства, эмоции) (Там же).

Многочисленные исследования с использованием каждодневного мониторинга эпизодов мечтательности и «ухода в свои мысли» в повседневной деятельности, а также лабораторные исследования показывают, что частота «ухода в свои мысли» увеличивается при переживании негативных эмоций (Hurlburt, 1980; McVay et al., 2009; Killingsworth, Gilbert, 2010). Эмоции могут служить для прерывания когнитивных 
систем, вовлеченных в текущую обработку сигналов, для того, чтобы обратиться к более важным личностным целям, переключить внимание на себя (Salovey, Mayer, 1990).

\section{Предполагаемые механизмы возникновения эпизодов «ухода в свои мысли»}

Упрощенно можно представить, что у человека есть два принципиально различных канала информации, поступающей в сознание, внешний (стимулы внешней среды) и внутренний (например, информация из эпизодической памяти). В ряде исследований было показано, что если «награда» за ориентацию на внешние стимулы велика (текущая стрессовая ситуация или иная ситуация, требующая повышенной настороженности), то человек может одновременно перерабатывать два канала без статистически значимых нарушений в восприятии внешней информации (Antrobus et al., 1966; Antrobus et al., 1967; Antrobus et al., 1970). Люди, склонные придавать высокую значимость внутренним переживаниям, своему состоянию, чаще уходят в свои мысли, что подтверждается показателями по шкале «Социальная интроверсия» (Миннесотский многоаспектный личностный опросник, MMPI) (Singer, Antrobus, 1963; Wagman, 1968).

Исторически предложено несколько объяснительных механизмов появления эпизодов «ухода в свои мысли» (Antrobus et al., 1970; McVay, Kane, 2010; Smallwood, Schooler, 2006; Schooler et al., 2011).

Согласно гипотезе отсоединения (decoupling hypothesis), «уход в свои мысли» использует те же когнитивные механизмы и ресурсы, что и обработка информации, связанной с текущей задачей. В рамках данного подхода «уход в свои мысли» - это активный когнитивный процесс. Он конкурирует с другими текущими когнитивными процессами за некоторый «общий центральный когнитивный оператор» с ограниченной емкостью или пул когнитивных ресурсов, включая ресурсы внимания (Antrobus et al., 1970; Smallwood, Schooler, 2006).

Дж.С. МакВей и М.Дж. Кейн выдвинули гипотезу нарушения исполнительного контроля, они утверждают, что «уход в свои мысли» представляет собой переключение внимания на отвлекающую информацию из-за ошибки в системе исполнительного контроля (McVay, Kane, 2010). При этом подразумевается, что «уход в свои мысли» является не активным, а скорее пассивным процессом психики, неким «холостым ходом», не потребляющим когнитивные ресурсы, в который сознание переходит при временном отсутствии управления со стороны исполнительного контроля.

В более поздних исследованиях Дж.У. Скулер с сотрудниками предложили гипотезу нарушения метапознания. В соответствии с этой гипотезой переключение внимания на эндогенно генерируемую информацию возникает из-за нарушения мониторинга и оценки содержания сознания (Schooler et al., 2011).

Таким образом, независимо от вопроса об ограниченности ресурсов, система когнитивного контроля играет основную роль в проблеме возникновения состояния «ухода в 
свои мысли» (Botvinick et al., 2001). Видимо, из-за неспособности когнитивного контроля удержать репрезентацию цели в рамках текущей деятельности происходит быстрое переключение всего когнитивного процесса на более личностно значимую цель. Многочисленные исследования говорят в пользу того, что в «дефолтной» сети мозга (т.е. сети, «активной по умолчанию», при отсутствии эксплицитной задачи) интегрируется информация о текущих важных для личности целях (образ себя, эпизодическая память, воспоминания с сенсорно-перцептивными деталями) (Christoff et al., 2009; Gusnard, Raichle, 2001; Knyazev et al., 2012). В каждый момент времени существует иерархия целей, которая управляет поведением (Carver, Scheier, 1999). Оценка целей по их значимости выражается в аффективном отношении, зависит от субъективно предполагаемой выгоды, которую субъект может получить от ориентации поведения на достижение той или иной цели. Далеко не всегда эксплицитная текущая деятельность обладает более высокой мотивационной значимостью, чем состояние «ухода в свои мысли».

Мотивационная значимость «ухода в свои мысли» складывается из двух суммирующихся составляющих. Во-первых, это мотивационная значимость конкретного содержания мыслей в данном состоянии (личностно значимые события в прошлом или будущем данного индивида). Вовторых, данное состояние, видимо, имеет собственную мотивационную значимость, входя в число неотъемлемых потребностей. Второй аспект предположительно сформировался в эволюции человека как полезный для выживания, так как обеспечивает анализ прошлого опыта и планирование будущих действий, отдавая максимум когнитивных ресурсов на эти процессы. Отсюда следует относительная непроизвольность наступления моментов «ухода в свои мысли», которые нередко возникают исподволь и которым трудно сопротивляться, подобно тому, как трудно противиться голоду, жажде или сонливости при соответствующем мотивационном состоянии.

Связь «ухода в свои мысли» с творчеством и креативностью (Singer, McCraven, 1961; Baird et al., 2012) может быть объяснена тем, что информация о целях, которые значимы для личности, подвергается межмодальному синтезу и претерпевает многократные перекомбинации, что может привести на уровне субъективного опыта к креативным решениям значимых для личности задач, активным творческим стратегиям поведения. Этот аспект феномена «ухода в свои мысли», видимо, является следствием и высшим развитием эволюционного смысла данного явления, который был рассмотрен выше и заключается в обработке личностно значимой информации вне контекста текущего времени, что дает мощный инструмент анализа информации.

\section{Связь феномена «ухода в свои мысли» с рефлексией, метапознанием (метасознанием) и полнотой осознания (mindfulness)}

В.П. Зинченко, сравнивая взгляды различных авторов на рефлексию, 
указывает на ее неоднородность. Фоновая рефлексия, недоступная сознанию, встречается даже в элементарных двигательных актах. Есть процессуальная фоновая рефлексия без Я. Эффекты такой рефлексии появляются по ходу действия. Она возникает 3-4 раза в секунду (Зинченко, 2010). Есть рефлексия в виде исследования понятий - она характерна для диалектического теоретического мышления. На рефлексии основываются оценка и контроль действий в деятельности (Зинченко, 2005). Продуктивное восприятие и мышление как творческий акт включает в себя процессы рефлексии и смыслообразования (Зинченко, 2010).

В.Д. Шадриков утверждает, что рефлексия выступает в жизни человека «важнейшим фактором овладения собственным поведением» (Шадриков, 2007, с. 213-220). Рефлексия позволяет человеку корректировать свою мыслительную деятельность и направлять в правильное русло для решения конкретных задач.

В рамках психологии «личностного смысла» рефлексия представляет собой «способность произвольного обращения человеком сознания на самого себя. Это понятие содержит в себе два принципиальных момента: механизм произвольного манипулирования идеальными содержаниями в умственном плане, основанный на переживании дистанции между своим сознанием и его интенциональным объектом... и направленность этого процесса на самого себя как на объект рефлексии» (Леонтьев и др., 2009, с. 146).

Согласно дифференциальной модели рефлексивности, существует три качественно различных формы рефлексии (Леонтьев, Осин, 2014):

1) интроспекция - фокус внимания переводится с внешней деятельности на внутренние состояния, переживания;

2) квазирефлексия - направление фокуса внимания на объекты, не имеющие отношения к актуальной жизненной ситуации, мечты, фантазии, воспоминания, «уход в посторонние размышления - о прошлом, будущем, о том, что было бы, если бы...»;

3) системная рефлексия - самодистанцирование, «взгляд на себя со стороны», позволяющий видеть одновременно «полюс субъекта и полюс объекта».

Этим трем состояниям в рамках дифференциальной модели рефлексивности противопоставляется направление внимания непосредственно на внешний интенциональный объект деятельности, что соответствует состоянию полного отсутствия рефлексии или арефлексии (Там же).

Понятие «метапознание» (также «метасознание») отчасти пересекается с понятием «рефлексия». Впервые термин «метапознание» (metacognition) ввел Дж. Флейвелл и определил его как способность анализировать собственные мыслительные стратегии и управлять своей познавательной деятельностью (Flavell, 1976).

Управление своим телом и содержанием своего сознания связано с поддержанием цели, планированием, контролем и обратной связью. Нервные процессы, поддерживающие репрезентацию актуальной 
информации в сетях мозга и руководящие целенаправленным поведением, обычно называют системой когнитивного контроля (Botvinick et al., 2001; Yeung et al., 2005). В литературе также встречается термин «исполнительный контроль», «исполнительные функции». Рефлексивные процессы (рефлексия и метапознание) могут представлять собой субъективное проявление части системы реализации когнитивного контроля, которая осуществляет связь между внешними и внутренними событиями, проверяя их на адекватность правилам текущей задачи.

Дж. Скулер схематически представляет взаимодействие сознательных, бессознательных и метасознательных процессов (Schooler, 2002). Сознание постоянно сопровождается неосознаваемым процессом мониторинга. Благодаря этому мониторингу осуществляется прослеживание изменений цели, выбор стратегии, модулируется содержание мыслей. Периодически могут возникать ситуации, когда необходимо пересмотреть содержание своего сознания, - ситуация отказа от цели, которая привлекает внимание, эмоциональная реакция и т.д. Метапознание - это повторная репрезентация содержания сознания, благодаря которой происходит его интерпретация и оценка. В связи с тем, что происходит повторное перекодирование информации, возникают несоответствия, которые необходимо учитывать при использовании самоотчетов: трудности с вербальным отображением невербального опыта, временно́е рассогласование. Связь рефлексивных процессов и когнитивного контроля обнаруживается и в развитии этих процессов в онтогенезе (Zelazo, 2004). В более поздних исследованиях Дж. Скулера и К. Кристофф с соавт. с применением фМРТ было обнаружено, что зоны мозга, ответственные за процессы метапознания и когнитивного контроля (передняя префронтальная кора, задняя поясная кора), также участвуют в генерации мыслей, не связанных с текущей задачей. Было сделано предположение, что возникает дополнительный поток информации, который занимает ресурсы когнитивного контроля и метапознания. Тот факт, что данные системы заняты альтернативным задаче потоком информации, может сделать их невосприимчивыми к детекции этого отклонения сознания (Christoff et al., 2009; Schooler et al., 2011). Таким образом, до настоящего времени роль процессов когнитивного контроля в генерации «ухода в свои мысли» и/или в предотвращении его возникновения недостаточно ясна, хотя связь между ними, несомненно, важна и заслуживает дальнейших исследований.

Рефлексия как фактор саморегуляции представляет собой механизм, при помощи которого рассматривается взаимоотношение субъекта и познания (в частности, выполнение задачи). Метапознание - это способ использования рефлексии для контроля над познанием (выполнением задачи). «Уход в свои мысли» - это переключение с текущего познания на альтернативный поток личностно значимой информации. С точки зрения дифференциальной модели рефлексивности те феномены, которые получили название квазирефлексии 
и интроспекции, по своим характеристикам похожи на феномен «ухода в свои мысли», так как отражают перенаправление внимания от текущей задачи, однако, чтобы различить эти понятия, мы предлагаем рассматривать рефлексию и метапознание как фактор преимущественно осознаваемой саморегуляции поведения, который направлен на успешное выполнение задачи, непосредственно стоящей перед человеком. «Уход в свои мысли» - это спонтанный, непроизвольный сбой внимания и нарушение саморегуляции в контексте текущего момента, которое зачастую приводит к нарушению текущей деятельности. С этой точки зрения - это два противоположных феномена, которые могут иметь под собой разные механизмы возникновения и поддержания. Однако в долговременной перспективе оба феномена адаптивны.

Клинический психолог К. Гермер (Germer, 2004) выводит определение полной осознанности, диалектически, сопоставляя с невнимательностью. Полнота осознания (mindfulness) - это состояние бодрствования, в котором человек полностью внимателен к тому, что происходит здесь и сейчас в отличие от невнимательности (mindlessness). Невнимательность - это поглощенность отвлеченными размышлениями и оценками происходящих событий. Согласно К. Гермеру, «полнота осознания в отличие от невнимательности фокусирует наше внимание на поставленной задаче. Когда мы в состоянии осознанности, наши мысли не запутываются в прошлом или будущем, мы не судим и не отвергаем то, что происходит с нами в данный момент. Мы присутствуем. Этот вид внимания способствует поднятию жизненной энергии и ясности мыслей, радости и уравновешенности. К счастью, этот навык можно развивать» (Там же, р. 25).

В состоянии полной осознанности можно выделить три составляющих компонента (Germer, 2004):

1. Осведомленность (awareness) это «фон» или «радар» сознания. Постоянный контроль внешней и внутренней среды.

2. Внимание (attention) - это процесс концентрации сознания в осведомленности. Обеспечение повышения чувствительности в ограниченном диапазоне опыта. Внимание постоянно выделяет «фигуры» из «фона» осведомленности, удерживая их в течение разных промежутков времени.

3. Припоминание (remembering) участвует в перенаправлении ускользающего внимания на осознание текущего опыта, контролируя рецептивную (безоценочную, констатирующую) форму восприятия действительности.

Поскольку стандартизованной процедуры исследования полноты осознания пока не существует и определение этого состояния нуждается в соблюдении научной строгости, то данные, полученные разными исследователями, трудно сопоставить между собой.

М.Д. Мразек рассматривает состояние полноты осознания как устойчивость к отвлекающим стимулам при выполнении текущей задачи (Mrazek et al., 2012). В современной психологической литературе в полноте осознания часто выделяется этот элемент (Brown, Ryan, 2003; 
Wallace, Shapiro, 2006). Испытуемые, предрасположенные к полноте осознания в повседневной жизни, и испытуемые после 10 минут практики концентрации реже отчитываются об эпизодах «ухода в свои мыли» (Mrazek et al., 2012).

В свете представленных данных «уход в свои мысли» и полнота осознания находятся в оппозиционных отношениях. С точки зрения эмоционального восприятия «уход в свои мысли» всегда связан с эмоциональным восприятием внутреннего эндогенного потока информации, в то время как полнота осознания - это безоценочное восприятие текущего опыта. Можно сказать, что полнота осознания связана с повышением саморегуляции и фиксации внимания на безоценочном восприятии событий внутри и вне тела, что может рассматриваться как конкретная когнитивная задача, а «уход в свои мысли» связан с нарушением ее выполнения.

\section{Заключение}

В современной психологической науке неоправданно мало внимания отводится изучению перехода от восприятия внешней информации и ситуации «здесь и сейчас» к внутренним, эндогенным процессам. Caмоосознание человека базируется на интеграции знаний о себе в настоящем, воспоминаний себя в прошлом и антиципации будущих событий. Происходит постоянное сравнение текущего опыта с личностными мотивами и ожиданиями.

«Уход в свои мысли» - неоднозначное, противоречивое состояние сознания. В отличие от рефлексив- ных процессов (рефлексия и метапознание) «уход в свои мысли» можно в узком смысле рассматривать как нарушение саморегуляции, отвлечение от поставленной задачи, которое в той или иной степени препятствуют успешному выполнению текущей деятельности.

Полнота осознания и «уход в свои мысли» находятся в оппозиционных отношениях с точки зрения устойчивости к отвлекающим стимулам при выполнении текущей задачи. Однако возникают значительные сложности при попытке четкого научного определения состояния полноты осознания.

Основная трудность в изучении феномена «ухода в свои мысли» заключается в том, что можно фиксировать наличие этого состояния, но не момент возникновения или его точную продолжительность. Благодаря современным технологическим достижениям, его можно исследовать более подробно, сочетая в работе самоотчеты испытуемых, поведенческие данные и данные нейровизуализации. Также весьма интересным представляется в данном контексте дальнейшее изучение функционирования «дефолтной» сети мозга.

В разные исторические периоды и в разных культурах люди по-разному относятся к мечтательности, которая неотъемлемо присутствует в психической жизни каждого человека. 3. Фрейд считал мечтателей инфантильными невротиками. В восточных культурах практики концентрации нацелены на развитие способности человека «быть здесь и сейчас», т.е. полностью погружаться в свою текущую деятельность и остановить уход в свои мысли. 
Современные исследования указывают на то, что уход в свои мысли при выполнении человеком какойлибо деятельности - очень распространенное явление. С одной стороны, в контексте выполнения текущей деятельности оно несет в себе риск совершения ошибок по причине «сбоев внимания»; в результате оно представляет собой угрозу при многих видах профессиональной деятельности, особенно при операторской деятельности.

Однако одновременно «уход в свои мысли» полезен и адаптивен в долговременной перспективе, обеспечивая оценку опыта из событий прошлого, планирование будущей деятельности и инкубацию творческих решений. Общим объединяющим фактором содержания «ухода в свои мысли» является высокая личностная значимость, которая задействует механизмы перераспределения когнитивных ресурсов. Значение когнитивного контроля в инициации и остановке эпизодов «ухода в свои мысли» требует дальнейшего пристального исследования.

\section{Литература}

Выготский, Л. С. (1982). Мышление и речь. В кн. В. В. Давыдов (Ред.), Проблемы общей психологии. Собрание сочинений в 6 томах (т. 2, с. 21-22). М.: Педагогика.

Гальперин, П. Я. (1998). К проблеме внимания. В кн. А. И. Подольский (Ред.), Психология как объективная наука (с. 415-425). М.: Изд-во «Институт практической психологии».

Зинченко, В. П. (2005). Готовность к мысли. К 75-летию В.В. Давыдова (1930-1998). Культурноисторическая психология, 2, 4-17.

Зинченко, В. П. (2010). Опыт думания о думании. К восьмидесятилетию В.В. Давыдова (19301998). Вопросы философии, 11, 75-91.

Леонтьев, Д. А., Лаптева, Е. М., Осин, Е. Н., Салихова, А. Ж. (2009). Разработка методики дифференциальной диагностики рефлексии. В кн. В. Е. Лепский (Ред.), Рефлексивные процессы и управление: Сборник материалов VII Международного симпозиума (с. 146). М.: Когито-Центр.

Леонтьев, Д. А., Осин, Е. Н. (2014). Рефлексия «хорошая» и «дурная»: от объяснительной модели к дифференциальной диагностике. Психология. Журнал Высшей школы экономики, 11(4), 110-135.

Шадриков, В. Д. (2007). Ментальное развитие человека. М.: Аспект Пресс.

Шадриков, В. Д. (2014). Мысль и познание. М.: Логос.

Antrobus, J. S., Coleman, R., \& Singer, J. L. (1967). Signal-detecting performance by subjects differing in predisposition to daydreaming. Journal of Consulting Psychology, 31(5), 487-491. doi:org/10.1037/h0024969

Antrobus, J. S., Singer, J. L., Goldstein, S., \& Fortgang, M. (1970). Mindwandering and cognitive structure. Transactions of the New York Academy of Sciences, 32, 242-252. doi:10.111 l/j.2164-0947.1970.tb02056.x

Antrobus, J. S., Singer, J. L., \& Greenberg, S. (1966). Studies in the stream of consciousness: experimental enhancement and suppression of spontaneous cognitive processes. Perceptual and Motor Skills, 23, 399-417. doi:10.2466/pms.1966.23. 2.399

Baird, B., Smallwood, J., Mrazek, M. D., Kam, J. W. Y., Franklin, M. S., \& Schooler, J. W. (2012). Inspired by distraction: mind wandering facilitates creative incubation. Psychological Science, 23(10), 1117-1122. doi:10.1177/0956797612446024 
Botvinick, M. M., Braver, T. S., Carter, C. S., Barch, D. M., \& Cohen, J. D. (2001). Conflict monitoring and cognitive control. Psychological Reviere, 108, 624-652. doi:org/10.1037/0033-295X.108.3.624

Brown, K. W., \& Ryan, R. M. (2003). The benefits of being present: Mindfulness and its role in psychological well-being. Journal of Personality and Social Psychology, 84, 822-848. doi:10.1037/00223514.84.4.822

Carver, C. S., \& Scheier, M. (1999). Themes and issues in the self-regulation of behavior. In R. S. Wyer (Ed.), Perspectives on behavioral self-regulation: Advances in social cognition (pp. 46-50). Mahwah, NJ: Erlbaum.

Christoff, K., Gordon, A. M., Smallwood, J., Smith, R., \& Schooler, J. W. (2009). Experience sampling during fMRI reveals default network and executive system contributions to mind wandering. PNAS: Proceedings of the National Academy of Sciences of the United States of America, 106, 87198724. doi:10.1073/pnas.0900234106

Flavell, J. H. (1976). Metacognitive aspects of problem solving. In L.B. Resnick (Ed.), The nature of intelligence (Ch. 12, pp. 231-235). Hillsdale, NJ: Erlbaum.

Germer, C. (2004). What is mindfulness? Insight Journal, 22, 24-29.

Gusnard, D. A., \& Raichle, M. E. (2001). Searching for a baseline: Functional imaging and the resting human brain. Nature Revieres Neuroscience, 2, 685-694. doi:10.1038/35094500

Hurlburt, R., T. (1980). Validation and correlation of thought sampling with retrospective measures. Cognitive Therapy and Research, 4(2), 235-238.

James, W. (1983). The stream of thought. In G. A. Miller (Ed.), The principles of psychology (pp. 150-192). Cambridge, MA/London: Harvard University Press.

Kaplan, R. L., Damme, I. V., \& Levine, L. J. (2012). Motivation matters: differing effects of pre-goal and post-goal emotions on attention and memory. Frontiers in Psychology, 3, 404. doi:10.3389/fpsyg.2012.00404

Killingsworth, M. A., \& Gilbert, D. T. (2010). A wandering mind is an unhappy mind. Science, 330, 932. doi:org/10.1126/science.1192439

Klinger, E., Grégoire, K. C, \& Barta, S. G. (1973). Physiological correlates of mental activity: Eye movements, alpha, and heart rate during imagining, suppression, concentration, search, and choice. Psychophysiology, 10, 471-477. doi:10.1111/j.1469-8986.1973.tb00534.x

Knyazev, G. G., Savostyanov, A. N., Volf , N. V., Liou, M., \& Bocharov, A. V. (2012). EEG correlates of spontaneous self-referential thoughts: A cross-cultural study. International Journal of Psychophysiology, 86, 173-181. doi:org/10.1016/j.ijpsycho.2012.09.002

Martin, L. L., \& Tesser, A. (1996). Some ruminative thoughts. In R. S. Wyer (Ed.), Ruminative thoughts: Advances in social cognition (Vol. 9, pp. 1-47). Hillsdale, NJ: Erlbaum.

McMillan, R. L., Kaufman, S. B., \& Singer, J. L. (2013). Ode to positive constructive daydreaming. Frontiers in Psychology, 4, 626. doi:org/10.3389/fpsyg.2013.00626

McVay, J. C., \& Kane, M. J. (2010). Does mind wandering reflect executive function or executive failure? Comment on Smallwood and Schooler (2006) and Watkins (2008). Psychological Bulletin, 136, 188-197. doi:org/10.1037/a0018298

McVay, J. C., Kane, M. J., \& Kwapil, T. R. (2009). Tracking the train of thought from the laboratory into everyday life: An experience-sampling study of mind wandering across controlled and ecological contexts. Psychonomic Bulletin and Review, 16(5), 857-863. doi:10.3758/PBR.16.5.857

Mrazek, M. D., Smallwood, J., \& Schooler, J. (2012). Mindfulness and mind-wandering: finding convergence through opposing constructs. Emotion, 12(3), 442-448. doi:10.1037/a0026678 
Rapaport, D. (1951). The conceptual model of psychoanalysis. Journal of Personality, 20(1), 56-81. doi:10.1111/j. 1467-6494.1951.tb01513.x

Rapaport, D. (1957). Cognitive structures. In M. M. Gill (Ed.), The collected papers of David Rapaport (pp. 631-664). New York: Basic Books.

Salovey, P., \& Mayer, J. D. (1990). Emotional intelligence. Imagination, Cognition and Personality, 9(3), 185-211. doi:10.2190/DUGG-P24E-52WK-6CDG

Schad, D. J., Nuthmann, A., \& Engbert, R. (2012). Your mind wanders weakly, your mind wanders deeply: Objective measures reveal mindless reading at different levels. Cognition, 125, 179-194. doi:org/10.1016/j.cognition.2012.07.004

Schooler, J. W. (2002). Re-representing consciousness: dissociations between experience and metaconsciousness. Trends in Cognitive Sciences, 6(8), 339-344. doi:10.1016/S1364-6613(02)01949-6

Schooler, J. W., Smallwood, J., Cristoff, K., Handy, T. C., Reichle, E. D., \& Sayette, M. A. (2011). Metaawareness, perceptual decoupling and the wandering mind. Trends in Cognitive Sciences, 15(7), 319-326. doi:10.1016/j.tics.2011.05.006

Singer, J. L. (1966). Daydreaming: An introduction to the experimental study of inner experience. New York: Crown Publishing Group/Random House.

Singer, J. L. (1993). Experimental studies of ongoing conscious experience. In G. R. Bock \& J. Mash (Eds.), Novartis Foundation Symposium. Experimental and theoretical studies of consciousness (Vol. 174, pp. 100-122). Chichester: Wiley.

Singer, J. L., \& Antrobus, J. S. (1963). A factor-analytic study of daydreaming and conceptually-related cognitive and personality variables. Perceptual and Motor Skills, 17, 187-209.

Singer, J. L., \& Antrobus, J. S. (1966). Imaginal processes inventory. New York: Center for Research in Cognition and Affect Graduate Center, City University of New York.

Singer, J. L., \& McCraven, V. G. (1961). Some characteristics of adult daydreaming. The Journal of Psychology: Interdisciplinary and Applied, 51(1), 151-164. doi:org/10.1080/00223980.1961.9916467

Singer, J. L., \& Schonbar, R. A. (1961). Correlates of daydreaming: a dimension of self-awareness. Journal of Consulting Psychology, 25(1), 1-6. doi:org/10.1037/h0048906

Smallwood, J. (2013). Distinguishing how from why the mind wanders: a process-occurrence framework for self-generated mental activity. Psychological Bulletin, 139(3), 519-535. doi:org/10.1037/a0O30010

Smallwood, J., \& Schooler, J. W. (2006). The restless mind. Psychological Bulletin, 132(6), 946-958. doi:org/10.1037/0033-2909.132.6.946

Speckens, A. E., Ehlers, A., Hackmann, A., Ruths, F. A., \& Clark, D. M. (2007). Intrusive memories and rumination in patients with post-traumatic stress disorder: A phenomenological comparison. Memory, 15(3), 249-257.

Vaitl, D., Birbaumer, N., Gruzelier, J., Jamieson, G. A., Kotchoubey, B., Kübler, A., ... Weiss, T. (2005). Psychobiology of altered states of consciousness. Psychological Bulletin, 131(1), 98-127. doi:org/10.1037/0033-2909.131.1.98

Wagman, M. (1968).The relationship of types of daydream behavior to selected MMPI scales. Psychiatry: Journal for the Study of Interpersonal Processes, 31(1), 84-89. doi:10.1521/00332747.1968.11023537

Wallace, B. A., \& Shapiro, S. (2006). Mental balance and well being: Building bridges between Buddhism and western psychology. American Psychologist, 61, 690-701. doi:10.1037/0003066X.61.7.690 
Watkins, E. R. (2008). Constructive and unconstructive repetitive thought. Psychological Bulletin, 134(2), 163-206. doi:org/10.1037/0033-2909.134.2.163

Werner, H., \& Wapner, S. (1949). Sensory-Tonic field theory of perception. Journal of Personality, 18(1), 88-107. doi:10.1111/j.1467-6494.1949.tb01235.x

Wolman, R. N., \& Kozmova, M. (2007). Last night I had the strangest dream: varieties of rational thought processes in dream reports. Consciousness and Cognition, 16, 838-849. doi:org/10.1016/j.concog.2006.09.009

Yeung, N., Nystrom, L. E., Aronson, J.A., \& Cohen, J. D. (2005). Between-task competition and cognitive control in task switching. The Journal of Neuroscience, 26(5), 1429-1438. doi:10.1523/JNEUROSCI.3109-05.2006

Zelazo, P. D. (2004). The development of conscious control in childhood. Trends in Cognitive Sciences, 8(1), 12-17. doi:10.1016/j.tics.2003.11.001

Zhiyan, T., \& Singer, J. L. (1997). Daydreaming styles, emotionality and the big five personality dimensions. Imagination, Cogition and Pesonality, 16, 399-414. doi:10.2190/ATEH-96EV-EXYX-2ADB

\title{
The Phenomenon of Mind-Wandering in the Continuum of Consciousness
}

Anna A. Lapina

Ph.D.-student, School of psychology HSE*; laboratory assistant, Research and study group of cognitive psychophysiology, HSE*

E-mail: alapina@hse.ru

\section{Boris V. Chernyshev}

Head of Research and study group of cognitive psychophysiology, HSE*; head of department, assistant professor, Department of Psychophysiology, School of psychology HSE*; Assistant professor, Department of higher nervous activity, Faculty of biology, Lomonosov Moscow State University**, Ph.D.

E-mail: bchernyshev@hse.ru

Address: * 20 Myasnitskaya str., Moscow, 101000, Russian Federation

** GSP-1, Leninskie Gory, Moscow, 119991, Russian Federation

\begin{abstract}
Mind-wandering is a specific state of consciousness, during which attention fully or partially switches from perception of external sensory information to inner mental processes. This state is largely spontaneous, and its content usually cannot be consciously controlled. Thoughts during mind wandering are generated by endogenous mental processes focusing on goals of high personal significance. These thoughts adversely interfere with processing of incoming information, thus leading to decreased efficacy of the ongoing activity, resulting in attentional lapses and errors. Dreaminess as a personality trait is directly related to mind wandering. Switching to the mindwandering state is related to competition between the motivational value of this process with the motivational value of current explicit activities, and it depends on cognitive (executive) control processes. The motivational value of mind wandering itself depends not only on the personal significance of the current thought content; this state appears also to be one of the basic human
\end{abstract}


needs providing humans with the analysis of past experience and planning future activities, and thus is highly advantageous in a long-term perspective. Mind-wandering is closely related to a number of other psychological phenomena such as reflection, metacognition (meta-consciousness), and mindfulness. The current review distinguishes between these phenomena and presents an approach to consider mind wandering a distinct process hallmarked by adverse effects on the ongoing activity.

Keywords: consciousness, continuum of consciousness, mind-wandering, attention, attentional lapses, daydreaming, reflection, metacognition, meta-consciousness, mindfulness.

\section{References}

Antrobus, J. S., Coleman, R., \& Singer, J. L. (1967). Signal-detecting performance by subjects differing in predisposition to daydreaming. Journal of Consulting Psychology, 31(5), 487-491. doi:org/10.1037/h0024969

Antrobus, J. S., Singer, J. L., Goldstein, S., \& Fortgang, M. (1970). Mindwandering and cognitive structure. Transactions of the New York Academy of Sciences, 32, 242-252. doi:10.111 l/j.2164-0947.1970.tb02056.x

Antrobus, J. S., Singer, J. L., \& Greenberg, S. (1966). Studies in the stream of consciousness: experimental enhancement and suppression of spontaneous cognitive processes. Perceptual and Motor Skills, 23, 399-417. doi:10.2466/pms.1966.23. 2.399

Baird, B., Smallwood, J., Mrazek, M. D., Kam, J. W. Y., Franklin, M. S., \& Schooler, J. W. (2012). Inspired by distraction: mind wandering facilitates creative incubation. Psychological Science, 23(10), 1117-1122. doi:10.1177/0956797612446024

Botvinick, M. M., Braver, T. S., Carter, C. S., Barch, D. M., \& Cohen, J. D. (2001). Conflict monitoring and cognitive control. Psychological Review, 108, 624-652. doi:org/10.1037/0033-295X.108.3.624

Brown, K. W., \& Ryan, R. M. (2003). The benefits of being present: Mindfulness and its role in psychological well-being. Journal of Personality and Social Psychology, 84, 822-848. doi:10.1037/00223514.84.4.822

Carver, C. S., \& Scheier, M. (1999). Themes and issues in the self-regulation of behavior. In R. S. Wyer (Ed.), Perspectives on behavioral self-regulation: Advances in social cognition (pp. 46-50). Mahwah, NJ: Erlbaum.

Christoff, K., Gordon, A. M., Smallwood, J., Smith, R., \& Schooler, J. W. (2009). Experience sampling during fMRI reveals default network and executive system contributions to mind wandering. PNAS: Proceedings of the National Academy of Sciences of the United States of America, 106, 87198724. doi:10.1073/pnas.0900234106

Flavell, J. H. (1976). Metacognitive aspects of problem solving. In L.B. Resnick (Ed.), The nature of intelligence (Ch. 12, pp. 231-235). Hillsdale, NJ: Erlbaum.

Galperin, P. Ya. (1998). K probleme vnimaniya [On the issue of attention]. In A. I. Podolskij (Ed.), Psikhologiya kak ob"ektivnaya nauka [Psychology as objective science] (pp. 415-425). Moscow: Institut Prakticheskoi Psikhologii.

Germer, C. (2004). What is mindfulness? Insight Journal, 22, 24-29.

Gusnard, D. A., \& Raichle, M. E. (2001). Searching for a baseline: Functional imaging and the resting human brain. Nature Reviewes Neuroscience, 2, 685-694. doi:10.1038/35094500 
Hurlburt, R., T. (1980). Validation and correlation of thought sampling with retrospective measures. Cognitive Therapy and Research, 4(2), 235-238.

James, W. (1983). The stream of thought. In G. A. Miller (Ed.), The principles of psychology (pp. 150-192). Cambridge, MA/London: Harvard University Press.

Kaplan, R. L., Damme, I. V., \& Levine, L. J. (2012). Motivation matters: differing effects of pre-goal and post-goal emotions on attention and memory. Frontiers in Psychology, 3, 404. doi:10.3389/fpsyg.2012.00404

Killingsworth, M. A., \& Gilbert, D. T. (2010). A wandering mind is an unhappy mind. Science, 330, 932. doi:org/10.1126/science.1192439

Klinger, E., Grégoire, K. C, \& Barta, S. G. (1973). Physiological correlates of mental activity: Eye movements, alpha, and heart rate during imagining, suppression, concentration, search, and choice. Psychophysiology, 10, 471-477. doi:10.1111/j.1469-8986.1973.tb00534.x

Knyazev, G. G., Savostyanov, A. N., Volf , N. V., Liou, M., \& Bocharov, A. V. (2012). EEG correlates of spontaneous self-referential thoughts: A cross-cultural study. International Journal of Psychophysiology, 86, 173-181. doi:org/10.1016/j.ijpsycho.2012.09.002

Leontiev, D. A., Lapteva, E. M., Osin, E. N., \& Salikhova, A. Zh. (2009). Razrabotka metodiki differentsial'noi diagnostiki refleksii [The development of the questionnaire of differential diagnostics of reflection]. In V. E. Lepskii (Ed.), Refleksionye protsessy i upravlenie: Sbornik materialov VII Mezhdunarodnogo simpoziuma [Reflective processes and management: Proceedings of VII International symposium] (pp. 145-150). Moscow: Kogito-Tsentr.

Leontiev, D. A., \& Osin, E. N. (2014). "Good" and "bad" reflection: From an explanatory model to differential assessment. Psychology. Journal of Higher School of Economics, 11(4), 110-135.

Martin, L. L., \& Tesser, A. (1996). Some ruminative thoughts. In R. S. Wyer (Ed.), Ruminative thoughts: Advances in social cognition (Vol. 9, pp. 1-47). Hillsdale, NJ: Erlbaum.

McMillan, R. L., Kaufman, S. B., \& Singer, J. L. (2013). Ode to positive constructive daydreaming. Frontiers in Psychology, 4, 626. doi:org/10.3389/fpsyg.2013.00626

McVay, J. C., \& Kane, M. J. (2010). Does mind wandering reflect executive function or executive failure? Comment on Smallwood and Schooler (2006) and Watkins (2008). Psychological Bulletin, 136, 188-197. doi:org/10.1037/a0018298

McVay, J. C., Kane, M. J., \& Kwapil, T. R. (2009). Tracking the train of thought from the laboratory into everyday life: An experience-sampling study of mind wandering across controlled and ecological contexts. Psychonomic Bulletin \& Reviere, 16(5), 857-863. doi:10.3758/PBR.16.5.857

Mrazek, M. D., Smallwood, J., \& Schooler, J. (2012). Mindfulness and mind-wandering: finding convergence through opposing constructs. Emotion, 12(3), 442-448. doi:10.1037/a0026678

Rapaport, D. (1951). The conceptual model of psychoanalysis. Journal of Personality, 20(1), 56-81. doi:10.1111/j. 1467-6494.1951.tb01513.x

Rapaport, D. (1957). Cognitive structures. In M. M. Gill (Ed.), The collected papers of David Rapaport (pp. 631-664). New York: Basic Books.

Salovey, P., \& Mayer, J. D. (1990). Emotional intelligence. Imagination, Cognition and Personality, 9(3), 185-211. doi:10.2190/DUGG-P24E-52WK-6CDG

Schad, D. J., Nuthmann, A., \& Engbert, R. (2012). Your mind wanders weakly, your mind wanders deeply: Objective measures reveal mindless reading at different levels. Cognition, 125, 179-194. doi:org/10.1016/j.cognition.2012.07.004

Schooler, J. W. (2002). Re-representing consciousness: dissociations between experience and metaconsciousness. Trends in Cognitive Sciences, 6(8), 339-344. doi:10.1016/S1364-6613(02)01949-6 
Schooler, J. W., Smallwood, J., Cristoff, K., Handy, T. C., Reichle, E. D., \& Sayette, M. A. (2011). Metaawareness, perceptual decoupling and the wandering mind. Trends in Cognitive Sciences, 15(7), 319-326. doi:10.1016/j.tics.2011.05.006

Shadrikov, V. D. (2007). Mental'noe razvitie cheloveka [Mental development of man]. Moscow: Aspekt Press.

Shadrikov, V. D. (2014). Mysl'i poznanie [Thought and cognition]. Moscow: Logos.

Singer, J. L. (1966). Daydreaming: An introduction to the experimental study of inner experience. New York: Crown Publishing Group/Random House.

Singer, J. L. (1993). Experimental studies of ongoing conscious experience. In G. R. Bock \& J. Mash (Eds.), Novartis Foundation Symposium. Experimental and theoretical studies of consciousness (Vol. 174, pp. 100-122). Chichester: Wiley.

Singer, J. L., \& Antrobus, J. S. (1963). A factor-analytic study of daydreaming and conceptually-related cognitive and personality variables. Perceptual and Motor Skills, 17, 187-209.

Singer, J. L., \& Antrobus, J. S. (1966). Imaginal processes inventory. New York: Center for Research in Cognition and Affect Graduate Center, City University of New York.

Singer, J. L., \& McCraven, V. G. (1961). Some characteristics of adult daydreaming. The Journal of Psychology: Interdisciplinary and Applied, 51(1), 151-164. doi:org/10.1080/00223980.1961.9916467

Singer, J. L., \& Schonbar, R. A. (1961). Correlates of daydreaming: a dimension of self-awareness. Journal of Consulting Psychology, 25(1), 1-6. doi:org/10.1037/h0048906

Smallwood, J. (2013). Distinguishing how from why the mind wanders: a process-occurrence framework for self-generated mental activity. Psychological Bulletin, 139(3), 519-535. doi:org/10.1037/a0O30010

Smallwood, J., \& Schooler, J. W. (2006). The restless mind. Psychological Bulletin, 132(6), 946-958. doi:org/10.1037/0033-2909.132.6.946

Speckens, A. E., Ehlers, A., Hackmann, A., Ruths, F. A., \& Clark, D. M. (2007). Intrusive memories and rumination in patients with post-traumatic stress disorder: A phenomenological comparison. Memory, 15(3), 249-257.

Vaitl, D., Birbaumer, N., Gruzelier, J., Jamieson, G. A., Kotchoubey, B., Kübler, A., ... Weiss, T. (2005). Psychobiology of altered states of consciousness. Psychological Bulletin, 131(1), 98-127. doi:org/10.1037/0033-2909.131.1.98

Vygotsky, L. S. (1982). Myshlenie i rech' [Thinking and speech]. In V. V. Davydov (Ed.), Problemy obshchei psikhologii. Sobranie sochinenii v 6 tomakh [Issues of general psychology. Collected works in 6 volumes] (Vol. 2, pp. 21-22). Moscow: Pedagogika.

Wagman, M. (1968).The relationship of types of daydream behavior to selected MMPI scales. Psychiatry: Journal for the Study of Interpersonal Processes, 31(1), 84-89. doi:10.1521/00332747.1968.11023537

Wallace, B. A., \& Shapiro, S. (2006). Mental balance and well being: Building bridges between Buddhism and western psychology. American Psychologist, 61, 690-701. doi:10.1037/0003066X.61.7.690

Watkins, E. R. (2008). Constructive and unconstructive repetitive thought. Psychological Bulletin, 134(2), 163-206. doi:org/10.1037/0033-2909.134.2.163

Werner, H., \& Wapner, S. (1949). Sensory-Tonic field theory of perception. Journal of Personality, 18(1), 88-107. doi:10.1111/j.1467-6494.1949.tb01235.x 
Wolman, R. N., \& Kozmova, M. (2007). Last night I had the strangest dream: varieties of rational thought processes in dream reports. Consciousness and Cognition, 16, 838-849. doi:org/10.1016/j.concog.2006.09.009

Yeung, N., Nystrom, L. E., Aronson, J.A., \& Cohen, J. D. (2005). Between-task competition and cognitive control in task switching. The Journal of Neuroscience, 26(5), 1429-1438. doi:10.1523/JNEUROSCI.3109-05.2006

Zelazo, P. D. (2004). The development of conscious control in childhood. Trends in Cognitive Sciences, 8(1), 12-17. doi:10.1016/j.tics.2003.11.001

Zhiyan, T., \& Singer, J. L. (1997). Daydreaming styles, emotionality and the big five personality dimensions. Imagination, Cogition and Pesonality, 16, 399-414. doi:10.2190/ATEH-96EV-EXYX-2ADB

Zinchenko, V. P. (2005). Readiness for thinking. Cultural-Historical Psychology, 2, 4-17.

Zinchenko, V. P. (2010). Opyt dumaniya o dumanii. K vos'midesyatiletiyu V.V. Davydova (1930-1998) [The experience of thinking about thinking. To the 80 years-jubilee of V.V. Davydov]. Voprosy Filosofii, 11, 75-91. 